TRANSACTIONS OF THE

AMERICAN MATHEMATICAL SOCIETY

Volume 355, Number 11, Pages 4365-4383

S 0002-9947(03)03292-6

Article electronically published on July 10, 2003

\title{
HYPERPLANE ARRANGEMENT COHOMOLOGY AND MONOMIALS IN THE EXTERIOR ALGEBRA
}

\author{
DAVID EISENBUD, SORIN POPESCU, AND SERGEY YUZVINSKY
}

\begin{abstract}
We show that if $X$ is the complement of a complex hyperplane arrangement, then the homology of $X$ has linear free resolution as a module over the exterior algebra on the first cohomology of $X$. We study invariants of $X$ that can be deduced from this resolution. A key ingredient is a result of Aramova, Avramov, and Herzog (2000) on resolutions of monomial ideals in the exterior algebra. We give a new conceptual proof of this result.
\end{abstract}

Let $X$ be the complement of a complex hyperplane arrangement $\mathcal{A}$. In this paper we study the singular homology $\mathrm{H}_{*}(X)$ as a module over the exterior algebra $E$ on the first singular cohomology $V:=\mathrm{H}^{1}(X)$ always with coefficients in a fixed field $K$. Our first main result (Section 11) asserts that $\mathrm{H}_{*}(X)$ is generated in a single degree and has a linear free resolution; this amounts to an infinite sequence of statements about the multiplication in the Orlik-Solomon algebra $\mathrm{H}^{*}(X)$. We also analyze other topological examples from the point of view of resolutions over the exterior algebra.

In Section 2 we study an invariant of an $E$-module $N$ called the singular variety, the algebraic subset of $V$ consisting of those elements $x$ whose annihilator in $N$ is not equal to $x N$. The singular variety is the same for $N$ and for $N^{*}$, and thus for the homology and cohomology of $X$. Aramova, Avramov and Herzog AAH show that the codimension of the singular variety (called by them the rank variety) gives the rate of growth of the free resolution of $N$. We compute the singular variety of $\mathrm{H}^{*}(X)\left(\right.$ or $\left.\mathrm{H}_{*}(X)\right)$ : it is a linear subspace of codimension equal to the number of central arrangements in an expression of $\mathcal{A}$ as a product of irreducible arrangements.

One way to use the linearity of the resolution of $\mathrm{H}_{*}(X)$ is through the BernsteinGel'fand-Gel'fand correspondence, which produces a graded module $F(\mathcal{A})$ over the symmetric algebra on $W:=V^{*}=\mathrm{H}_{1}(X)$ corresponding to $X$, whose associated sheaf $\mathcal{F}(\mathcal{A})$ is supported on the singular variety. In Section 3 we compute this invariant of $X$ (really an invariant of the intersection poset of the arrangement). We compute some homological invariants of $F(\mathcal{A})$ and we use its properties to show that the cones over a generic hyperplane arrangement may be characterized as the

\footnotetext{
Received by the editors April 1, 2001.

2000 Mathematics Subject Classification. Primary 15A75, 52C35, 55N45; Secondary 55N99, $14 \mathrm{Q} 99$.

The first two authors are grateful to the NSF for support during the preparation of this work. The authors would like to thank the Mathematical Sciences Research Institute in Berkeley for its support while part of this paper was being written.
} 
arrangements for which the defining ideal (the Orlik-Solomon ideal) of $\mathrm{H}^{*}(X)$ also has a linear free resolution.

If $e \in E_{1}$, then $e$ corresponds to a local system on the complement of the hyperplane arrangement $\mathcal{A}$ (see Esnault-Schechtman-Viehweg [ESV], Yuzvinsky [Yu], and Libgober-Yuzvinsky [LY] Section 4]). If we set $A=\mathrm{H}^{*}(X)$ and $\mathrm{Ann}_{A} e=$ $\{a \in A \mid e a=0\}$, then the homology

$$
\mathrm{H}(e, A):=\left(\operatorname{Ann}_{A} e\right) / e A
$$

of the complex defined through multiplication by $e$ is the cohomology of $X$ with supports in that local system, for $e$ subject to a certain mild genericity condition (Esnault-Schechtman-Viehweg [ESV]). In Section 4 we show how to compute $\mathrm{H}(e, A)$. In terms of the sheaf $\mathcal{F}(\mathcal{A})$ we have

$$
\mathrm{H}(e, A)=\operatorname{Tor}_{*}^{\mathcal{O}_{\mathbb{P}, e}}\left(\kappa(e), \mathcal{F}(\mathcal{A})_{e}\right)
$$

where $\mathbb{P}=\mathbb{P}\left(E_{1}^{*}\right)$ is the projective space of one-dimensional subspaces of $E_{1}$, and $\kappa(e)$ is the residue field of the local ring $\mathcal{O}_{\mathbb{P}, e}$ at the point corresponding to $e$. (We prove in Theorem 4.1 a more general corresponding result, for arbitrary modules $A$ with linear injective resolution.) It follows, for example, that when $e$ is singular on $A$ the module $\mathrm{H}(e, A)$ has nonzero components in every degree up to the codimension of $F(\mathcal{A})$, cf. Theorem 4.1 (b). This generalizes Theorem 4.1 (i) of Yuzvinsky [Yu]; see also Libgober and Yuzvinsky [LY].

A key ingredient in the proof of our main theorem is the theorem of Aramova, Avramov, and Herzog $\overline{\mathrm{AAH}}$ (later improved by Römer $\overline{\mathrm{Rö}}$ ) relating the resolutions of square-free monomial ideals (and some more general modules) over symmetric and exterior algebras. This allows us to apply the results on resolutions and Alexander duality due to Eagon and Reiner [ER. The proof given by Aramova, Avramov and Herzog depends on an intricate computation. In Section 5 we offer a conceptual description of the relationship which leads to a transparent proof.

We are pleased to acknowledge the essential role of the computer algebra system written by Grayson and Stillman GS] in the genesis of this paper: It was only through "playing" with this program that we were led to guess at the main result (Theorem 1.1) and most of the other results were checked for plausibility before we looked for proofs.

Notation. Throughout this paper, $\mathcal{A}$ will denote an essential affine complex hyperplane arrangement, that is, a set of $n$ affine hyperplanes in $\mathbb{C}^{\ell}$ whose intersection poset has rank $\ell$. We will denote the complement of the union of the hyperplanes in $\mathcal{A}$ by $X$. We denote by $K$ an arbitrary field.

We use the notation in Orlik and Terao [OT. In particular, we write $A:=A(\mathcal{A})$ for the Orlik-Solomon algebra of $\mathcal{A}$, isomorphic to the singular cohomology of $X$ with coefficients in $K$. The vector space $E_{1}=V=\mathrm{H}^{1}(X)$ has basis $e_{1}, \ldots, e_{n}$ corresponding to the hyperplanes of $\mathcal{A}$. Writing $E$ for the exterior algebra on $E_{1}$ we have $A=\mathrm{H}^{*}(X)=E / I$ where $I \subset E$ is the Orlik-Solomon ideal generated by the elements

$$
\partial\left(e_{i_{1}} \wedge \cdots \wedge e_{i_{t}}\right)=\sum_{j}(-1)^{j} e_{i_{1}} \wedge \cdots \wedge e_{i_{j-1}} \wedge \widehat{e_{i_{j}}} \wedge e_{i_{j+1}} \wedge \cdots \wedge e_{i_{t}}
$$

for which $\left\{H_{i_{1}}, \ldots, H_{i_{t}}\right\}$ is a minimal linearly dependent set of hyperplanes of $\mathcal{A}$, and the monomials $e_{i_{1}} \wedge \cdots \wedge e_{i_{t}}$ for which $\left\{H_{i_{1}}, \ldots, H_{i_{t}}\right\}$ have empty intersection. For all this see Orlik and Solomon $\mathrm{OS}$. 
We grade $E$ by taking the elements of $V$ to have degree 1 (this is the opposite convention from that of Eisenbud and Schreyer [ES]). The homology module $\mathrm{H}_{*}(X)$ is dual to $E / I$, and thus is graded in negative degrees. We denote its component of degree $-p$ by $\mathrm{H}_{p}(X)(p \geq 0)$.

We will write $\chi(\mathcal{A},-)$ for the characteristic polynomial of the arrangement $\mathcal{A}$. For our purposes $\chi$ may be defined by the relation

$$
\chi(\mathcal{A}, t)=t^{\ell} \pi(A,-1 / t),
$$

where $\pi$ is the Poincaré polynomial of $X$, that is,

$$
\pi(t)=\sum_{j} \operatorname{dim}_{K} \mathrm{H}^{j}(X) t^{j}
$$

see Orlik and Terao [OT Definition 2.52 and Theorem 3.68].

If $A$ is a skew commutative algebra, we write $A\langle e\rangle$ and $A[t]$ to denote the skewcommutative algebra obtained by adjoining a variable of degree 1 or 2 respectively; thus $A[t]$ is an ordinary polynomial ring on one commuting variable over $A$, while if $E$ is the exterior algebra of $V$, then $E\langle e\rangle$ is the exterior algebra of $V \oplus K e$.

\section{THE COHOMOLOGY OF HYPERPLANE ARRANGEMENTS}

Theorem 1.1. The minimal free resolution of $\mathrm{H}_{*}(X)$, regarded as a module over the exterior algebra $E=\wedge\left(\mathrm{H}^{1}(X)\right)$ by means of the cap product, has the form

$$
\mathbf{F}: \cdots \rightarrow E^{\beta_{2}}(\ell-2) \rightarrow E^{\beta_{1}}(\ell-1) \rightarrow E^{\beta_{0}}(\ell) \rightarrow \mathrm{H}_{*}(X) \rightarrow 0 .
$$

The ranks $\beta_{i}$ may be computed from the formula

$$
\sum_{i=0}^{\infty} \beta_{i} t^{i}=(-1)^{\ell} \frac{\chi(\mathcal{A}, t)}{(1-t)^{n}}
$$

In general we will say that a graded $E$-module $M$ has a linear resolution if $M$ is generated in a single degree $s$ and has free resolution with $d^{\text {th }}$ syzygy module generated in degree $s+d$; the theorem asserts that $H_{*}(X)$ has a linear resolution with $s=-\ell$.

We can interpret the statement that a module of the form $E^{\beta_{0}}(\ell)$ can map onto $\mathrm{H}_{*}(X)$ in more familiar language:

Corollary 1.2. An element $c \in \mathrm{H}^{*}(X)$ is annihilated by the (cup) product with every element of $\mathrm{H}^{1}(X)$ if and only if $c \in \mathrm{H}^{\ell}(X)$.

Proof. Because $E^{\beta_{0}}(\ell)$ maps onto $\mathrm{H}_{*}(X)$, we see that $\mathrm{H}_{*}(X)$ is generated as an $E$-module by $\mathrm{H}_{\ell}(X)$. In particular, we recover the well-known fact that $\mathrm{H}^{j}(X)=$ $\left(\mathrm{H}_{j}(X)\right)^{*}=0$ for $j>\ell$, so that every element of $\mathrm{H}^{\ell}(X)$ is annihilated by $\mathrm{H}^{1}(X)$.

Conversely, let $c \in \mathrm{H}^{*}(X)$ be annihilated by $\mathrm{H}^{1}(X)$. The Orlik-Solomon description shows that $\mathrm{H}^{*}(X)$ is generated as an algebra by $\mathrm{H}^{1}(X)$. So $c$ is annihilated by $\mathrm{H}^{+}$, the ideal of elements of positive degree in $\mathrm{H}^{*}(X)$. In particular, $c \cdot\left(\mathrm{H}^{+} \cdot \mathrm{H}_{*}(X)\right)=0$. Because $\mathrm{H}_{*}(X)$ is generated by $\mathrm{H}_{\ell}(X)$ we have $\mathrm{H}^{+} \cdot \mathrm{H}_{*}(X)=\sum_{j<\ell} \mathrm{H}_{j}(X)$. Since each $\mathrm{H}_{j}(X)$ is dual to $\mathrm{H}^{j}(X)$ by the multiplication pairing, it follows that $c \in \mathrm{H}^{\ell}(X)$.

For the proof of Theorem 1.1 it is convenient to reduce to the central case. Recall that an arrangement is central if the intersection of its hyperplanes is nonempty. Given a (not necessarily central) arrangement $\mathcal{A}$ of $n$ hyperplanes in $\mathbb{C}^{\ell}$, we can 
projectivize and add the hyperplane at infinity to get an arrangement in $\mathbb{P}_{\mathbb{C}}^{\ell}$; the affine cone over this arrangement is a central arrangement $\mathcal{B}=c \mathcal{A}$ of $n+1$ hyperplanes in $\mathbb{C}^{\ell+1}$, called the cone over $\mathcal{A}$. Conversely, given a central arrangement $\mathcal{B}$ of $n+1$ hyperplanes in $\mathbb{C}^{\ell+1}$, and a chosen hyperplane $H$ in it, we may form the corresponding arrangement of $n+1$ hyperplanes in projective $\ell$-space. Removing $H$, we get a (not necessarily central) arrangement $\mathcal{A}=d \mathcal{B}$ of $n$ hyperplanes in $\mathbb{C}^{\ell}$, which we call the deconing of $\mathcal{B}$ with respect to $H$. For example, $\mathcal{A}$ is the deconing of $c \mathcal{A}$ with respect to the "new" hyperplane.

The Orlik-Solomon algebras of $\mathcal{B}$ and $d \mathcal{B}$ are closely related. Topologically, the complement of the projective arrangement associated to $\mathcal{B}$ is the same as the complement of the arrangement associated to any of the deconings of $\mathcal{B}$; thus the complement of $\mathcal{B}$ is a $\mathbb{C}^{*}$-bundle over the complement of any of the deconings of $\mathcal{B}$. It follows that the cohomology algebra of any deconing is canonically isomorphic to the cohomology algebra of the complement of $\mathcal{B}$ modulo a degree 1 form. The following result gives this identification algebraically. For this it is convenient to factor the Orlik-Solomon relations as products of linear forms:

Proposition 1.3. Suppose $\mathcal{B}=\left\{H_{0}, \ldots, H_{n}\right\}$ is a central hyperplane arrangement, with Orlik-Solomon ideal $I$ in the exterior algebra $E=K\left\langle e_{0}, \ldots, e_{n}\right\rangle$ whose generators $e_{i}$ correspond to the hyperplanes $H_{i}$. Let $E^{\prime}$ be the subalgebra generated by the differences $e_{i}-e_{j}$. Let $I^{\prime} \subset E^{\prime}$ be the ideal generated by

$$
\left\{\left(e_{i_{1}}-e_{i_{2}}\right)\left(e_{i_{2}}-e_{i_{3}}\right) \cdots\left(e_{i_{s}-1}-e_{i_{s}}\right) \mid H_{i_{1}}, \ldots, H_{i_{s}} \text { are linearly dependent }\right\} .
$$

The Orlik-Solomon ideal of $\mathcal{B}$ is $I=I^{\prime} E$, and $E / I \cong\left(E^{\prime} / I^{\prime}\right)\left\langle e_{j}\right\rangle$ for any $j$. Furthermore, if $\mathcal{A}$ is the deconing of $\mathcal{B}$ with respect to $H_{j}$, then the Orlik-Solomon algebra of $\mathcal{A}$ is $E /\left(I+\left(e_{j}\right)\right) \cong E^{\prime} / I^{\prime}$.

Proof. One checks directly that $\left(e_{i_{1}}-e_{i_{2}}\right) \cdots\left(e_{i_{s}-1}-e_{i_{s}}\right)=(-1)^{s} \partial\left(e_{i_{1}} \wedge \cdots \wedge e_{i_{s}}\right)$. It follows that $I=I^{\prime} E$. The rest of the statements are consequences.

Proof of Theorem 1.1. To prove that the resolution of $\mathrm{H}_{*}(X)$ is linear, we first reduce to the central case. By Proposition 1.3 the Orlik-Solomon algebra of $c \mathcal{A}$ is $A\left\langle e_{0}\right\rangle=A \otimes_{K} K\left\langle e_{0}\right\rangle$ as skew-commutative algebras, and it follows that the free resolution of the homology of the complement of $c \mathcal{A}$ is deduced from that of $\mathcal{A}$ by tensoring over $K$ with $K\left\langle e_{0}\right\rangle$. In particular, one is linear if and only if the other one is, and we may assume that $\mathcal{A}$ is central to begin with.

With respect to the lexicographic order on the monomials of $E$, taking $e_{i}<e_{j}$ if $i<j$, the initial (largest) terms of the generators for the Orlik-Solomon ideal (as given in the introduction) are

$$
\begin{gathered}
\left\{e_{i_{1}} \wedge \cdots \wedge e_{i_{s}} \mid i_{1}<\cdots<i_{s},\left\{H_{i_{1}}, \ldots, H_{i_{s}}\right\}\right. \text { is an independent set of } \\
\text { hyperplanes, and there exists } i_{0}<i_{1} \text { such that } \\
\left.\left\{H_{i_{0}}, \ldots, H_{i_{s}}\right\} \text { is dependent }\right\} .
\end{gathered}
$$

The subsets that appear in this expression are exactly the broken circuits of $c \mathcal{A}$. By Björner Bjö1], the monomials that are not divisible by broken circuits are a basis for $A$. It follows that the generators of $I$ given in the introduction form a Gröbner basis. Consequently the initial ideal of $I$ is the ideal $I_{0}$ generated by the 
monomials in the display. $I_{0}$ is the broken circuit ideal of the matroid defined by the dependence relations among the hyperplanes of $\mathcal{A}$.

From the general theory of Gröbner bases (as, for example, in Eisenbud Ei] where the completely parallel theory is treated for ideals in a polynomial ring) we see that $I_{0}$ is a flat degeneration of $I$. More formally, there is an ideal $I_{t} \subset$ $K[t] \otimes_{K} E$ such that the algebra $K[t] \otimes_{K} E / I_{t}$ is free (and thus flat) over $K[t]$, and $I_{0}:=(I+(t)) /(t) \subset K[t] \otimes_{K} E /(t)=E$ is the initial ideal in $(I)$, while for $0 \neq a \in K$ we have $I_{a}:=(I+(t-a)) /(t-a) \subset K[t] \otimes_{K} E /(t-a)=E$ is conjugate to $I$ by a linear automorphism of $E$.

The module structure on $\mathrm{H}_{*}(X)$ comes from the identification $\mathrm{H}_{*}(X)=$ $\left.\operatorname{Hom}_{K}\left(\mathrm{H}^{*}(X), K\right)\right)$. So $\mathrm{H}_{*}(X)$ degenerates flatly to $M_{0}=\operatorname{Hom}_{K}\left(E / I_{0}, K\right)$. More formally, the module $M=\operatorname{Hom}_{K[t]}\left(E / I_{t}, K[t]\right)$ is free (and thus flat) over $K[t]$, and has special fiber $M /(t) M \cong M_{0}$, whereas for $a \neq 0$ the fiber $M_{a}:=M /(t-a) M$ is conjugate to $\mathrm{H}_{*}(X)$ by an automorphism of $E$.

The first statement of Theorem 1.1 amounts to saying that the $k^{\text {th }}$ graded component, $\operatorname{Tor}_{j}^{E}\left(\mathrm{H}_{*}(X), K\right)_{k}$, vanishes for all $j>0$ and $k \neq \ell-j$. The vanishing of any one of these vector spaces is an open condition in flat families. So it suffices to show that $M_{0}=\operatorname{Hom}_{K}\left(E / I_{0}, K\right)$ satisfies the conditions of Theorem 1.1

The algebra $E$ is Gorenstein (injective as a module over itself) with socle in degree $n$. So $M_{0}=\operatorname{Hom}_{K}\left(E / I_{0}, K\right)=\operatorname{Hom}_{E}\left(E / I_{0}, E\right)(n)$ as $E$-modules. On the other hand, $\operatorname{Hom}_{E}\left(E / I_{0}, E\right)$ may be identified with the annihilator $J_{0}$ of $I_{0}$, and we see that it suffices to show that $J_{0}$ has free resolution of the form

$$
\mathbf{F}(-n): \cdots \rightarrow E^{\beta_{2}}(\ell-2-n) \rightarrow E^{\beta_{1}}(\ell-1-n) \rightarrow E^{\beta_{0}}(\ell-n) \rightarrow J_{0} \rightarrow 0
$$

Since $I_{0}$ is generated by monomials, so is the ideal $J_{0}$. Following Aramova, Avramov, and Herzog [AAH] (see also Section 5 below for more details) we let ${ }_{S} I_{0}$ and ${ }_{S} J_{0}$ be the ideals of $S=K\left[e_{1}, \ldots, e_{n}\right]$ generated by the monomials corresponding to the generators of $I_{0}$ and $J_{0}$, respectively, so that ${ }_{S} I_{0}$ and ${ }_{S} J_{0}$ are square-free monomial ideals of $S$. Aramova, Avramov, and Herzog [AAH] show that $J_{0}$ has a free resolution as above with $d^{\text {th }}$ syzygies generated in degree $d+n-\ell$ if and only if ${ }_{S} J_{0} \subset S$ has a resolution with this same property; for another proof, see Section 5 below.

Any square-free monomial ideal $J$ corresponds to a simplicial complex $\Delta(J)$. Since $I_{0}$ and $J_{0}$ are annihilators of one another in $E$, the simplicial complex $\Delta\left(I_{0}\right)$ is the Alexander dual of $\Delta\left(J_{0}\right)$; that is, the faces of $\Delta\left(J_{0}\right)$ are the complements of the nonfaces of $\Delta\left(I_{0}\right)$. By Eagon and Reiner [ER], ${ }_{S} J_{0}$ has a (linear) resolution as above if and only if ${ }_{S} I_{0}$ has codimension $n-\ell$ and $S /\left({ }_{S} I_{0}\right)$ is Cohen-Macaulay or, in combinatorial terms, the simplicial complex $\Delta\left(I_{0}\right)$ is Cohen-Macaulay of dimension $\ell-1$. (See also the later papers of Terai [Te], Bayer-Charalambous-Popescu [BCP], Mustaţă $[\mathrm{Mu}$, and Yanagawa [Ya] for more sophisticated versions of this result.)

It was observed by Hochster [Ho1] and Stanley St2] that the Cohen-Macaulay property of a simplicial complex follows from a simpler geometric property called shellability; see also Stanley [St1, Theorem 2.5] and Bruns-Herzog [BH1, Theorem 5.1.13]. It is known that the simplicial complex corresponding to the broken circuits of a matroid of rank $\ell$ is shellable of dimension $\ell-1$ (Provan $[\mathrm{Pr}$; see also Björner Bjö2 7.4.2(ii) and 7.4.3] and his reference Billera and Provan [BP]), concluding the proof of the first statement. 
In order to prove the second statement we note that, from the given resolution,

$$
\begin{aligned}
\pi\left(\mathrm{H}_{*}(X), t\right) & =\sum_{i}(-1)^{i} \pi\left(E^{\beta_{i}}(\ell-i), t\right) \\
& =\sum_{i}(-1)^{i} \beta_{i} t^{-\ell+i}(1+t)^{n} .
\end{aligned}
$$

On the other hand, since homology and cohomology are dual, $\pi\left(\mathrm{H}_{*}(X), t\right)=$ $\pi(A, 1 / t)=(-1)^{\ell} \chi(A,-t) / t^{\ell}$, whence the desired formula.

In general, we do not know how to write the free resolution of the Orlik-Solomon ideal explicitly; this seems to be an interesting problem.

Remark 1.4. Here are a few other topological examples treated from the point of view of resolutions over the exterior algebra:

a) Perhaps the most familiar topological spaces with cohomology generated in degree one are compact orientable surfaces. If $Y$ is an orientable compact connected surface of genus $g>0$, then the homology $\mathrm{H}_{*}(Y)$ does not satisfy Theorem 1.1 though it comes close: by Poincaré duality the homology $\mathrm{H}_{*}(Y)$ is isomorphic as a module over $E=\wedge \mathrm{H}^{1}(Y)$ to $\mathrm{H}^{*}(Y)$, which has relations of degree $>1$. However, if we write $\mathrm{H}^{*}(Y)=E / I$, then one can check that (with respect to any monomial order on $E$ ) the initial ideal of $I$ is the square-free stable ideal consisting of all but the last monomial of degree 2 in E. By Aramova, Herzog, and Hibi $\underline{\mathrm{AHH}}$. Corollary 2.5], the initial ideal, and with it $I$ itself, has linear resolution. It follows that the minimal free resolution of the homology module has the form

$$
\mathbf{F}: \cdots \rightarrow E^{\beta_{3}}(-2) \rightarrow E^{\beta_{2}}(-1) \rightarrow E^{\left(\begin{array}{c}
2 g \\
2
\end{array}\right)-1} \rightarrow E(2) \rightarrow \mathrm{H}_{*}(Y) \rightarrow 0 .
$$

b) A result analogous to Theorem 1.1 holds for the homology module of an essential arrangement of real subspaces of codimension two in $\mathbb{R}^{2 n}$ with even-dimensional intersections. In this case the cohomology ring of the complement has again the shape of an Orlik-Solomon algebra. However, in contrast with the complex case, it is not determined merely by the intersection lattice, but requires the knowledge of extra information on sign patterns (computed as determinants of linear relations, or as linking numbers in the sense of knot theory); see Björner-Ziegler [BZ] and Ziegler [Zi] for details.

c) The complements of codimension two subspace arrangements in $\mathbb{R}^{4}$ are equivalent to the link complements obtained by intersecting them with the three-sphere $S^{3}$. More generally, consider the case of an arbitrary tame link $L=\bigcup_{i=1}^{n} L_{i}$ in $S^{3}$, and let $X$ be the compact manifold with boundary that is the complement of a tubular neighborhood of $L$. Alexander duality gives $\operatorname{dim} \mathrm{H}^{1}(X)=n, \operatorname{dim} \mathrm{H}^{2}(X)=n-1$ and $\mathrm{H}^{\geq 3}(X)=0$. More explicitly, let $e_{i} \in \mathrm{H}^{1}(X)$ be the dual of the meridian of the $i^{\text {th }}$ boundary component, and let $f_{i, j} \in \mathrm{H}^{2}(X)$ be the Alexander dual of the (relative) homology class of an $\operatorname{arc} \gamma_{i, j}$ connecting the $i^{\text {th }}$ and $j^{\text {th }}$ components of the boundary. The elements $e_{i}$ form a basis of $\mathrm{H}^{1}(X)$ and (with the conventions $f_{i, i}=0$ and $\left.f_{i, j}=-f_{j, i}\right)$ the $f_{i, j}$ generate $\mathrm{H}^{2}(X)$.

A Mayer-Vietoris argument shows that the cohomology ring of $X$ has a presentation

$$
\mathrm{H}^{*}(X)=\wedge V^{\prime} /\left(e_{i} \wedge e_{j}-l_{i, j} f_{i, j}, f_{i, j}+f_{j, k}+f_{k, i}, e_{k} \wedge f_{i, j}, f_{i, j} \wedge f_{k, l}\right),
$$


where $V^{\prime}=\mathrm{H}^{1}(X) \oplus \mathrm{H}^{2}(X)$, the numbers $i$ and $j$ run from 1 to $n$, and $l_{i, j}:=$ $\operatorname{lk}\left(L_{i}, L_{j}\right)$ is the linking number of $L_{i}$ and $L_{j}$. In particular, the cohomology algebra $\mathrm{H}^{*}(X)$ depends only on the linking numbers (for most of this, see Milnor [Mi]).

Let $G$ be the graph whose vertices are the components $L_{i}, i=1, \ldots, n$, and where two vertices $L_{i}$ and $L_{j}$ are connected by an edge if their linking number $l_{i, j}$ is nonzero. Assume that $G$ is connected and the ground field has characteristic 0 . The given relations then suffice to eliminate all the $f_{i, j}$, and it follows that $\mathrm{H}^{*}(X)$ is generated in degree 1 (see also Massey-Traldi $[\mathrm{MT}$, Theorem 1 and Proposition 4.1], or Matei-Suciu [MS]).

Under these hypotheses, the cohomology ring behaves very nicely:

Theorem 1.5. If $G$ is connected and the ground field has characteristic 0 , then both the homology module $\mathrm{H}_{*}(X)$ and the presentation ideal $I$ of the cohomology ring $\mathrm{H}^{*}(X)$ have linear free resolutions over the exterior algebra $E=\wedge \mathrm{H}^{1}(X)$.

Sketch of proof. With these hypotheses the presentation ideal $I \subset E$ is generated by the monomials $e_{i} \wedge e_{j}$, where $i$ and $j$ are vertices not connected by an edge in $G$, together with elements $\sum_{k}\left(1 / l_{i_{k}, i_{k+1}}\right) e_{i_{k}} \wedge e_{i_{k+1}}$, where the sum is over a cycle in the graph $G$. In particular, $E / I$ is a quotient ring of the (exterior algebra) StanleyReisner ring of the graph $G$, regarded as a 1-dimensional simplicial complex on the vertex set $\left\{e_{1}, \ldots, e_{n}\right\}$.

Now suppose we have chosen a spanning tree $T$ of the connected graph $G$, and a total order on the edges of $G$. Recall that an edge $e \in G \backslash T$ is called externally active in $T$ if it is the largest edge in the unique cycle $C_{e}$ contained in $T \cup\{e\}$. It is a standard fact that for each enumeration of the edges of $G$ (say corresponding to the choice of a monomial order in $E$ ) there exists a spanning tree $T_{0}$ of $G$ such that every edge of $G$ not in $T_{0}$ is externally active in $T_{0}$ (see Bollobás [Bo proof of Theorem 10, p. 351 and Exercise 8, p. 372]). Since the cycles $C_{e}$ form a basis of the cycle space of $G$ (see, for example, Bollobás [Bo proof of Theorem 9, p. 53]), it follows that the ideal $I$ has an initial ideal $I_{0}$, which is the Stanley-Reisner ideal of the chosen spanning tree $T_{0}$ in $G$.

The fact that the Stanley-Reisner ideal $I_{0}$ has a linear resolution follows from Hochster's formula for the Betti numbers of a square-free monomial ideal (see Hochster [Ho2] or, for an exposition, Stanley [St1]) since any subcomplex of a tree is a forest, which is acyclic in all positive homological degrees. The linearity of the injective resolution of $\mathrm{H}^{*}(X)$ follows from the fact that $T$ is a Cohen-Macaulay simplicial complex as in the proof of Theorem 1.1.

\section{The Singular VARIETy OF AN ORLIK-SOlOMON Algebra}

An element $x \in V=E_{1}$ is said to be singular on a module $M$ if the set of elements of $M$ annihilated by $x$ is not the same as $x M$. The set $\operatorname{sing}(M)$ of singular elements is an algebraic subset of $V$ called the singular variety $\operatorname{sing}(M)$ of $M$; see Aramova, Avramov, and Herzog [AAH] for a discussion (but note that the term rank variety is used in place of singular variety). These authors prove, among other things, that the dimension of $\operatorname{sing}(M)$ is the complexity of $M$, defined as the exponent of growth of the Betti numbers of $M$. This complexity plays, for modules over an exterior algebra, a role analogous to that of the projective dimension for modules over a polynomial ring. In this section we will compute the singular variety of the OrlikSolomon algebra $A$ of an arrangement $\mathcal{A}$. It follows at once from the definition that 
the singular variety of a module $M$ is the same as that of $\operatorname{Hom}_{K}(M, K)$; so this also gives the singular variety of $\mathrm{H}_{*}(X)$.

Recall that the product $\mathcal{A}_{1} \times \mathcal{A}_{2}$ of arrangements $\mathcal{A}_{i}$ in $\mathbb{C}^{\ell_{i}}$ is the arrangement $\mathcal{A}$ in $\mathbb{C}^{\ell_{1}+\ell_{2}}$ consisting of the hyperplanes $H \times \mathbb{C}^{\ell_{2}}$ for $H \in \mathcal{A}_{1}$ and the hyperplanes $\mathbb{C}^{\ell_{1}} \times H$ for $H \in \mathcal{A}_{2}$. Any arrangement can be expressed uniquely as the product of irreducible arrangements. The following well-known remark shows that to compute the singular variety of the Orlik-Solomon algebra as a module over $E$, it suffices to treat the irreducible case:

Proposition 2.1. The Orlik-Solomon algebra of a product $\mathcal{A}=\mathcal{A}_{1} \times \mathcal{A}_{2}$ of two arrangements is given by $A(\mathcal{A})=A\left(\mathcal{A}_{1}\right) \otimes_{K} A\left(\mathcal{A}_{2}\right)$, the tensor product in the category of graded skew-commutative $K$-algebras. Thus $\operatorname{sing}(A(\mathcal{A}))=\operatorname{sing}\left(A\left(\mathcal{A}_{1}\right)\right) \times$ $\operatorname{sing}\left(A\left(\mathcal{A}_{2}\right)\right)$.

Proof. A minimal dependent set of hyperplanes in $\mathcal{A}$, or a minimal set with empty intersection, comes from a similar set either in $\mathcal{A}_{1}$ or in $\mathcal{A}_{2}$, proving the first statement. The second follows because $A(\mathcal{A})_{1}$ is the direct sum of the corresponding spaces for $\mathcal{A}_{1}$ and $\mathcal{A}_{2}$. A linear form $x=\left(x_{1}, x_{2}\right)$ is singular for $A(\mathcal{A})$ if $x_{i}$ is singular on $A\left(\mathcal{A}_{i}\right)$ for both $i=1,2$.

The main result of this section is:

Theorem 2.2. Let $\mathcal{A}$ be an irreducible hyperplane arrangement with Orlik-Solomon algebra $A$ and elements $e_{i} \in V:=A_{1}$ corresponding to the hyperplanes of $\mathcal{A}$.

a) If $\mathcal{A}$ is noncentral, then the singular variety of $A$ is $V$.

b) If $\mathcal{A}$ is central, then the singular variety of $A$ is the hyperplane spanned by the elements $e_{i}-e_{j}$.

Proof. If the singular variety of the Orlik-Solomon algebra $A$ of an arrangement $\mathcal{A}$ does not contain an element $e \in V$, then $A$ is a free module over the subring $K[e] / e^{2}$. It follows that the Poincaré polynomial $1+t$ of $K[e] / e^{2}$ divides the Poincaré polynomial $\pi(A, t)$ of $A$.

On the other hand, Crapo $\mathrm{Cr}$ (see also Schechtman-Terao-Varchenko STV Sect. 2]) shows that if $\mathcal{B}$ is an irreducible central arrangement with deconing $\mathcal{A}$, then

$$
\pi(A(\mathcal{B}), t) /(1+t)_{\mid t=-1} \neq 0
$$

It follows that in this case the singular variety of $A(\mathcal{A})$ contains everything of degree 1. In particular, if $\mathcal{A}$ is an irreducible noncentral arrangement, we may apply this remark to $\mathcal{B}=c \mathcal{A}$. Part $a$ ) now follows from Proposition 1.3.

If now $\mathcal{B}$ is an irreducible central arrangement, then the formula $A(\mathcal{B})=A(d \mathcal{B})[e]$ from Proposition 1.3 implies that the singular variety of $A(\mathcal{B})$ is equal to the singular variety of $E^{\prime} / I^{\prime} \cong A(d \mathcal{B})$; that is, it consists of precisely the elements of $V^{\prime}$ as required.

From Theorem 2.2 and Proposition 2.1 we get the general case:

Corollary 2.3. The singular variety of the Orlik-Solomon algebra $A$ of any arrangement $\mathcal{A}$ is a linear space of $V$ given in dual coordinates to the canonical basis $\left\{e_{H}\right\}_{H \in \mathcal{A}}$ by the system of equations

$$
\sum_{H \in \mathcal{A}_{j}} x_{H}=0
$$


for each central factor $\mathcal{A}_{j}$. In particular, the codimension of the singular variety of $A$ equals the number of central factors in an irreducible decomposition of $\mathcal{A}$.

Example 2.4. A central arrangement $\mathcal{A}$ in $\mathbb{C}^{\ell}$ is called generic if no set of $\ell$ or fewer hyperplanes of $\mathcal{A}$ is dependent. Analogously, a noncentral arrangement is called generic if every set of $\ell+1$ or fewer hyperplanes meet transversely (in particular, they do not meet if the number of hyperplanes is $\ell+1$ ). In the generic noncentral case it follows immediately from the definition that the Orlik-Solomon ideal $I$ is the $(\ell+1)^{\text {st }}$ power $\mathfrak{m}^{\ell}$ of the maximal ideal $\mathfrak{m}$ of $E$. From Proposition 1.3 it follows from this that in the generic central case the Orlik-Solomon ideal is the $\ell^{\text {th }}$ power of the maximal ideal of the subalgebra $E^{\prime}$ generated by the differences $e_{i}-e_{j}$ of the generators of $E$.

The homology module $\mathrm{H}_{*}(X)$ is, as for every arrangement, given by

$$
\mathrm{H}_{*}(X)=\operatorname{Hom}_{K}(E / I, K) \cong \operatorname{Hom}_{E}(E / I, E(n))=\left(0:_{E} I\right)(n),
$$

the $n^{\text {th }}$ twist of the annihilator of $I$. If $I=\mathfrak{m}^{\ell}$, then $(0: E I)(n)=\mathfrak{m}^{n-\ell+1}$. An explicit computation of the resolution of this ideal is given in terms of Schur functors in Eisenbud and Schreyer [ES, Corollary 5.3]; in particular, the resolution is linear.

\section{The module $F(\mathcal{A})$}

Let $W=V^{*}=\mathrm{H}_{1}(X)$ be the dual vector space to $V$, and let $S=\operatorname{Sym}(W)$ be the symmetric algebra of $W$, a polynomial ring over $K$.

As usually stated, the Bernstein-Gel'fand-Gel'fand correspondence (BGG) is an isomorphism between the derived category of bounded complexes of coherent sheaves on $\mathbb{P}\left(V^{*}\right)$ and the derived category of bounded complexes of finitely generated graded modules over $E=\wedge V$. But if one examines the proof, one can extract a functor $\mathbf{R}$ from the category of graded modules over $S$ and the category of linear free complexes over $E$, and also a functor $\mathbf{L}$ from the category of graded $E$-modules to the category of linear free complexes over $S$. These functors are equivalences of categories; see Eisenbud and Schreyer [ES, Proposition 2.1].

Starting with a graded $E$-module $P$, the corresponding complex $\mathbf{L}(P)$ over $S$ is

$$
\cdots \rightarrow S \otimes P_{i} \rightarrow S \otimes P_{i+1} \rightarrow \cdots
$$

with differential $1 \otimes p \mapsto \sum x_{i} \otimes e_{i} p$, where $x_{i}$ and $e_{i}$ are dual bases of $W$ and $V$. Starting with a graded $S$-module $M$ the corresponding complex $\mathbf{R}(M)$ over $E$ is

$$
\cdots \rightarrow \operatorname{Hom}_{K}\left(E, M_{i}\right) \rightarrow \operatorname{Hom}_{K}\left(E, M_{i+1}\right) \rightarrow \cdots,
$$

with differential defined similarly.

Starting from a hyperplane arrangement $\mathcal{A}$, we consider the injective resolution of $A$ as an $E$-module. Recall that since $E$ is Gorenstein, injective resolutions over $E$ are simply the duals (with respect to $E$ or to $K$ ) of free resolutions. Thus the injective resolution of $A$ is the $K$-dual of the free resolution of $\mathrm{H}_{*}(X)$. By Theorem 1.1, this free resolution, and with it the injective resolution of $A$, is linear.

Thus we may define $F(\mathcal{A})$ to be the graded $S$-module that is mapped by $\mathbf{R}$ to the injective resolution of $A$ as an $E$-module. The reason for choosing the injective resolution over the free resolution in the definition of $F(\mathcal{A})$ is to make $F(\mathcal{A})$ finitely generated. 
The following result, which is Theorem 3.7 of Eisenbud and Schreyer [ES] allows us to derive some basic properties of $F(\mathcal{A})$ :

Theorem 3.1. If $M$ is a graded $S$-module and $P$ is a finitely generated graded E-module, then $\mathbf{L}(P)$ is a free resolution of $M$ if and only if $\mathbf{R}(M)$ is an injective resolution of $P$.

Corollary 3.2. $F(\mathcal{A})$ is generated over $S$ in degree $\ell$ and has linear free resolution equal to $\mathbf{L}(A)$. In particular,

a) $F(\mathcal{A})$ has projective dimension $\ell$ and $\operatorname{Ext}_{S}^{\ell}(F(\mathcal{A}), S)=K$.

b) The support of $F(\mathcal{A})$ is a linear space whose codimension is the number of central arrangements in an irreducible decomposition of $\mathcal{A}$.

c) The Hilbert function of $F(\mathcal{A})$ is

$$
\sum_{i=0}^{\infty} \operatorname{dim}_{K}\left(F(\mathcal{A})_{i}\right) t^{i}=(-1)^{\ell} \frac{\chi(\mathcal{A}, t)}{(1-t)^{n}} .
$$

Proof. By Theorem 1.1 the injective resolution of $A$ over $E$, which is dual to the free resolution of $H_{*}(X)$, is linear. By Theorem 3.1.

$$
\mathbf{L}(A): \quad 0 \rightarrow S \otimes_{K} A_{0} \rightarrow \cdots \rightarrow S \otimes_{K} A_{\ell} \rightarrow F(\mathcal{A}) \rightarrow 0
$$

is a (linear) free resolution of $F(\mathcal{A})$, proving the first statement and computing the projective dimension.

$a$ ): The degree 0 and 1 parts of $A$ coincide with those of $E$; thus the left-hand terms of the resolution above are the same as those in $\mathbf{L}(E)$, the Koszul complex. This allows us to compute the Ext in part a).

$b)$ : Aramova, Avramov and Herzog [AAH] show in general that the singular variety of an $E$-module $P$ is the support of the $S=\operatorname{Ext}_{E}^{*}(K, K)$-module $\operatorname{Ext}_{E}^{*}(P, K)$, which is the same (since $E$ is Gorenstein) as the support of the module $\operatorname{Ext}_{E}^{*}(K, P)$. By Eisenbud and Schreyer [ES, Proposition 2.3] this is the module $F(\mathcal{A})$.

c): Knowing the free resolution of $F(\mathcal{A})$ allows us to compute its Hilbert series, just as in the proof of Theorem 1.1

Example 3.3. If $\mathcal{A}$ is a generic noncentral arrangement of $n$ hyperplanes in $K^{\ell}$, then $A$ is $E / \mathfrak{m}^{\ell+1}$. Thus the free resolution of $F(\mathcal{A})$ is a truncation of the Koszul complex, and $F(\mathcal{A})$ is isomorphic to the $(v-\ell)^{\text {th }}$ syzygy module of the trivial $S$-module $K$.

We have already seen that if $\mathcal{A}$ is a generic noncentral arrangement, then the Orlik-Solomon ideal of $\mathcal{A}$ is a power of the maximal ideal of $E$, and thus has a linear free resolution. We will show that this property characterizes generic arrangements and their cones. We begin with a general result characterizing deformations of powers of the maximal ideal:

Theorem 3.4. Let $I \subset E$ be an ideal in the exterior algebra. Both $I$ and $(E / I)^{*}$ admit linear free resolutions if and only if I reduces to a power of the maximal ideal modulo some (respectively any) maximal E/I regular sequence of linear forms of E.

Proof. If $f_{1}, \ldots, f_{s} \in E_{1}$ is a regular sequence on $E / I$, then $I$ and $(E / I)^{*}$ $=\operatorname{Hom}_{K}(E / I, K)$ are also free over $K\left\langle f_{1}, \ldots, f_{s}\right\rangle$. The freeness of $E / I$ over $K\left\langle f_{1}, \ldots, f_{s}\right\rangle$ implies that the image of $I$ in $E /\left(f_{1}, \ldots, f_{s}\right)$ is isomorphic to $I /\left(f_{1}, \ldots, f_{s}\right) I$, and also that the dual of $E /\left(I+\left(f_{1}, \ldots, f_{s}\right)\right)$ is $(E / I)^{*} \otimes_{E}$ 
$E /\left(f_{1}, \ldots, f_{s}\right)$. Thus the minimal free resolutions of $I$ and $(E / I)^{*}$ are linear if and only if the minimal resolutions of $I /\left(f_{1}, \ldots, f_{s}\right) I$ and $\left(E /\left(I+\left(f_{1}, \ldots, f_{s}\right)\right)\right)^{*}$ are linear, and it follows from Eisenbud and Schreyer [ES, Section 5] that if the image of $I$ in $E /\left(f_{1}, \ldots, f_{s}\right)$ is a power of the maximal ideal, then the minimal free resolutions of $I$ and $(E / I)^{*}$ are linear.

To prove the converse, the argument given above reduces to our showing, in the case where the singular variety of $E / I$ is $V$, that if the resolutions of $I$ and $(E / I)^{*}$ are linear, then $I$ is itself a power of the maximal ideal.

Our hypothesis implies, in particular, that the module $(E / I)^{*}$ is generated in a single degree. It follows by Nakayama's Lemma and duality that the socle of $E / I$ (the annihilator in $E / I$ of $\mathfrak{m}$ ) is generated in a single degree, say degree $s$. Thus $I_{j}=E_{j}$ for $j>s$, and it suffices to show that $I_{j}=0$ for $j \leq s$.

By Theorem 3.1, both $\mathbf{L}(E / I)$ and $\mathbf{L}\left(I^{*}\right)$ are free resolutions; let $F$ be the module whose resolution is $\mathbf{L}(E / I)$. By Aramova, Avramov, and Herzog AAH its support is the singular variety of $E / I$, that is, $V$.

Duality (into $K$ ) over the exterior algebra gives an exact sequence $0 \rightarrow(E / I)^{*} \rightarrow$ $E^{*} \rightarrow I^{*} \rightarrow 0$. Taking duals commutes with the functor $\mathbf{L}$ (up to shifts). So we get an exact sequence of complexes $0 \rightarrow \mathbf{L}(E / I)^{*} \rightarrow \mathbf{L}(E)^{*} \rightarrow \mathbf{L}\left(I^{*}\right) \rightarrow 0$, where now the duals denote $\operatorname{Hom}_{S}(-, S)$. The homology of $\mathbf{L}(E / I)^{*}$ at $S \otimes_{K}\left((E / I)_{s}\right)^{*}$ is $\operatorname{Ext}_{S}^{0}(F, S)$, which is nonzero because $F$ has support $V$. It follows from the exact sequence that $\mathbf{L}\left(I^{*}\right)$ has nonzero homology at the term $S \otimes\left(I_{s+1}\right)^{*}$. Since $\mathbf{L}\left(I^{*}\right)$ is a resolution, this must be the last term of the complex - that is, $I_{j}=0$ for $j \leq s$, as required.

Example 3.5. The ideals characterized in Theorem 3.4 include powers of the maximal ideal in subalgebras generated by linear forms (this will be the case for cones over hyperplane arrangements) but also many that are not of this form. Here is the simplest concrete example: Let

$$
I:=(a b+c d, a c, b c) \subset E:=K\langle a, b, c, d\rangle .
$$

It is easy to check that the three given quadrics form a Gröbner basis with respect to any order with $a b>c d$. Since $d$ is a regular element on $E$ modulo the initial ideal $(a b, a c, b c)$, it follows that $d$ is regular on $E / I$. It is evident that $I$ reduces modulo $d$ to the square of the maximal ideal. To see that $I$ is not the square of the maximal ideal of any exterior subalgebra on 3 variables, note that the quadrics in 3 variables are all of rank 2 , where $I$ contains an element of rank 4 (here the rank is defined via the identification between elements of $E_{2}$ and skew-symmetric $4 \times 4$ matrices).

Corollary 3.6. The Orlik-Solomon ideal of $\mathcal{A}$ admits a linear free resolution over $E$ if and only if $\mathcal{A}$ is obtained by successively coning a generic noncentral arrangement.

Proof. We have already seen that the property holds for generic noncentral arrangements. If $I$ is the Orlik-Solomon ideal of $\mathcal{A}$, then the Orlik-Solomon ideal of $c \mathcal{A}$ in $E\left[e_{0}\right]$ is $I E\left[e_{0}\right]=I \otimes_{E} E\left[e_{0}\right]$, which has free resolution obtained from that of $I$ by tensoring with $E\left[e_{0}\right]$; in particular, the linearity is not affected.

Deconing $\mathcal{A}$ as many times as possible, it now suffices to show that if $\mathcal{A}$ is noncentral and $I$ has a linear resolution, then $\mathcal{A}$ is generic. Since $\mathcal{A}$ is noncentral, it can have no central factors in its irreducible decomposition, and thus the singular variety of $\mathcal{A}$ is the whole of the vector space $V$ of linear forms. 
The theorem now follows from a more general result. Recall from Aramova, Avramov, and Herzog [AAH] that a sequence of elements $f_{1}, \ldots, f_{s} \in E_{1}$ is called a regular sequence on an $E$-module $M$ if $M$ is free over $K\left\langle f_{1}, \ldots, f_{s}\right\rangle$ or, equivalently, if the annihilator of $f_{i}$ in $M /\left(f_{1}, \ldots, f_{s}\right) M$ is $f_{i} M /\left(f_{1}, \ldots, f_{s}\right) M$ for every $i$. In this case the minimal free resolution of $M /\left(f_{1}, \ldots, f_{s}\right) M$ over $E /\left(f_{1}, \ldots, f_{s}\right)$ is obtained by reducing the minimal $E$-free resolution of $M$ modulo $\left(f_{1}, \ldots, f_{s}\right)$. The length of any maximal regular sequence on $M$ is equal to the codimension of the singular variety of $M$ in $V$.

Remark 3.7. Theorem 3.4 is actually equivalent to the Theorem of Horrocks that characterizes the bundle $\Omega_{\mathbb{P}(W)}^{i}(i)$ as the unique indecomposable sheaf $\mathcal{F}$ such that the only nonzero intermediate cohomology of any twist of $\mathcal{F}$ is $\mathrm{H}^{i}(\mathcal{F})=K$. To see this one uses the correspondence between powers of the maximal ideal of $E$ and the twisted exterior powers of the cotangent sheaf $\Omega_{\mathbb{P}(W)}^{i}(i)$, as well as the relation between resolutions over $E$ and cohomology of sheaves on $\mathbb{P}(W)$, all explained in Eisenbud and Schreyer [ES].

\section{How SINGULAR IS A SINGULAR ELEMENT?}

We keep the notation $W=V^{*}$ and $S=\operatorname{Sym}(W)$ from the last section. Let $N$ be a graded $E$-module, such as $\mathrm{H}^{*}(X)$. Denote the homology of $N \stackrel{e}{\rightarrow} N \stackrel{e}{\rightarrow} N$ by $\mathrm{H}(e, N)$; that is, $\mathrm{H}(e, N)=\left(\operatorname{Ann}_{N} e\right) / e N$, so that $\mathrm{H}(e, N) \neq 0$ if and only if $e$ is in the singular variety of $N$. In general, it is not easy to say in which degrees the homology $\mathrm{H}(e, N)$ will occur, but if $N$ has a linear injective resolution and socle in one degree, say degree 0 , as is the case of $\mathrm{H}^{*}(X)(l)$, then the following result shows that $\mathrm{H}(e, N) \neq 0$ if and only if $e: N_{1} \rightarrow N_{0}$ fails to be surjective, or equivalently if and only if $e$ annihilates a degree 0 element of $N^{*}$.

Theorem 4.1. Let $N$ be a finitely generated $E$-module with socle in degree 0 having a linear injective resolution, and let $F$ be the $S$-module such that the resolution of $N$ is $\mathbf{R}(F)$. For any $e \in V$ we have

a) For each $i$,

$$
\mathrm{H}(e, N)_{i}=\operatorname{Tor}_{i}^{\mathcal{O}_{\mathbb{P}, e}}\left(\kappa(e), \mathcal{F}_{e}\right),
$$

where $\kappa(e)$ denotes the residue class field of $\mathbb{P}=\mathbb{P}(W)$ at $e$, and $\mathcal{F}_{e}$ denotes the stalk at $e$ of the sheaf $\mathcal{F}$ on $\mathbb{P}$ corresponding to the module $F$.

b) $\mathrm{H}(e, N)_{i} \neq 0$ if and only if $i$ is between 0 and the projective dimension of $\mathcal{F}_{e}$

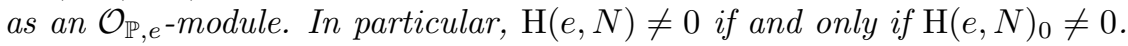

c) Suppose $e \in \operatorname{sing}(N)=X$ is a generic point of the component of $X$ in which it lies. If the codimension of $X \subset \mathbb{P}$ at $e$ is $c$, then $\mathrm{H}(e, N)_{i} \neq 0$ precisely for $0 \leq i \leq c$.

Problem 4.2. Characterize, in terms of the arrangement, the subset of $\operatorname{sing}\left(\mathrm{H}^{*}(X)\right)$ consisting of those $e$ for which the projective dimension of $\mathcal{F}(\mathcal{A})_{e}$ is greater than the codimension of the largest component of $\operatorname{sing}\left(\mathrm{H}^{*}(X)\right)$ on which $e$ lies. See Yuzvinsky [Yu, Falk [Fa], Cohen-Suciu [CS] and Libgober-Yuzvinsky [LY] for related computations.

Proof. Everything follows from the formula given in $a$ ). To prove this formula, recall that if $t \in W$ is any element outside $e^{\perp}$, then $\mathcal{F}_{e}$ may be represented as the degree 0 part of the localization, at $\left(e^{\perp}\right)$, of the module $F\left[t^{-1}\right]$. Thus if we write $R=S\left[t^{-1}\right]_{0}$, then the Tor that we are concerned with may be represented 
as $\operatorname{Tor}_{i}^{R}\left(R /\left(e^{\perp}\right)_{0}, F\left[t^{-1}\right]_{0}\right)$. Since localizing and taking the degree 0 part are both exact functors, we may rewrite this as $\operatorname{Tor}_{i}^{S}\left(S /\left(e^{\perp}\right)\left[t^{-1}\right], F\left[t^{-1}\right]\right)_{0}$. By Theorem 3.1. $\mathbf{L}(N)$ is a free resolution of $F$, and thus $\operatorname{Tor}_{i}^{S}\left(S /\left(e^{\perp}\right)\left[t^{-1}\right], F\left[t^{-1}\right]\right)_{0}$ is the $i^{\text {th }}$ homology of $\mathbf{L}(N) \otimes_{S} R_{e}$, where $R_{e}=K\left[t, t^{-1}\right]$ has $S$-module structure coming from the map $S \rightarrow K\left[t, t^{-1}\right]$ derived from $e: W \rightarrow K=K t$. But $\mathbf{L}(N) \otimes_{S} R_{e}$ is nothing but the complex

$$
\cdots \stackrel{t \otimes e}{\longrightarrow} R_{e} \otimes_{K} N_{i-1} \stackrel{t \otimes e}{\longrightarrow} R_{e} \otimes_{K} N_{i} \stackrel{t \otimes e}{\longrightarrow} R_{e} \otimes_{K} N_{i+1} \stackrel{t \otimes e}{\longrightarrow} \cdots
$$

whose degree 0 part is

$$
\cdots \stackrel{t \otimes e}{\longrightarrow} K t^{-i-1} \otimes_{K} N_{i-1} \stackrel{t \otimes e}{\longrightarrow} K t^{-i} \otimes_{K} N_{i} \stackrel{t \otimes e}{\longrightarrow} K t^{-i+1} \otimes_{K} N_{i+1} \stackrel{t \otimes e}{\longrightarrow} \cdots,
$$

a complex computing $\mathrm{H}(e, N)_{i}$.

Example 4.3. Each part of Theorem 4.1 fails for a module whose resolution is linear for $d$ steps, no matter how large $d$ is. To see this, note first that if we have an exact sequence

$$
0 \rightarrow N^{\prime} \rightarrow F \rightarrow N \rightarrow 0
$$

such that $F$ is free, then the homology under multiplication by $e$ satisfies $\mathrm{H}\left(e, N^{\prime}\right)=$ $\mathrm{H}(e, N)(1)$, Thus a module whose linear syzygy chain ends at a certain point cannot have a minimal generator annihilated by a linear form! Perhaps the simplest example is the dual of the $d^{\text {th }}$ module of syzygies of $E /(a b)$, where $a, b$ are independent linear forms.

Restating part of this in the case of a hyperplane arrangement, we get:

Corollary 4.4. An element $e \in V$ is singular for $A$ (or equivalently for $\mathrm{H}_{*}(X)$ ) if and only if there is a nonzero element of $\mathrm{H}_{\ell}(X)$ annihilated by $e$.

Proof. We have shown that $\mathrm{H}^{*}(X)$ has socle in degree $\ell$ (note that the signs are opposite to those of Eisenbud and Schreyer [ES]) and linear injective resolution. Thus $N=\mathrm{H}^{*}(X)(\ell)$ satisfies the hypothesis of Theorem 4.1 Thus $e$ is singular for $N$ if and only if $e: H^{\ell-1}(X) \rightarrow H^{\ell}(X)$ fails to be surjective, or, dually, $e$ : $H_{\ell}(X) \rightarrow H_{\ell-1}(X)$ fails to be injective.

\section{Syzygies of MONOMial IDEALS IN THE EXTERIOR ALgEBRA}

In this section we give a conceptual description and proof of the correspondence between free resolutions of certain modules over exterior and symmetric algebras first proved by Aramova, Avramov, and Herzog [AAH] and Römer [Rö]. The main idea is an isomorphism between certain subcategories of the categories of modules over these two algebras. Our approach provides a simple explanation for the shape of the formula relating the corresponding multigraded Betti numbers (Corollary 5.7).

Let $V$ be an $n$-dimensional vector space over the field $K$, with basis $x_{1}, \ldots, x_{n}$. We will denote by $S=\operatorname{Sym}(V)$ the symmetric algebra over $V$, which we identify with the ring of polynomials over $K$ in the $n$ variables $x_{1}, \ldots, x_{n}$, and by $E=\Lambda(V)$ the exterior algebra of the vector space $V$. Both of these algebras have a natural $\mathbb{Z}^{n}$ grading in which each monomial (product of the $x_{i}$ ) generates a homogeneous component. (Note that in earlier sections we wrote $S=\operatorname{Sym}(W)$, where $W$ was the dual of $V$. Since we have explicitly chosen a basis of $V$, we may identify $V$ with $W$. 
We say that a $\mathbb{Z}^{n}$-graded module $M$ over $E$ or $S$ is square-free if it admits a free presentation $F \rightarrow G \rightarrow M \rightarrow 0$ where each generator of $F$ and $G$ has the degree of a square-free monomial. Note that the presentation map $F \rightarrow G$ is represented by a matrix whose entries are scalars times monomials. Examples include the StanleyReisner rings $S / I$ where $I$ is an ideal generated by square-free monomials, but also such things as the cokernel of the matrix

$$
\left(\begin{array}{cc}
x_{0} & 0 \\
-x_{1} & x_{1} \\
0 & -x_{2}
\end{array}\right)
$$

the canonical module of the cone over 3 points in the plane.

There is a one-to-one correspondence between square-free modules over $S$ and over $E$ obtained by interpreting the presentations as matrices over $S$ or over $E$; we will write ${ }_{S} M$ and ${ }_{E} M$ for the two.

We can describe the correspondence of resolutions in a simple way as follows:

Start from a free resolution of a square-free module ${ }_{S} M$. Replace each free module in the resolution by a module made from the sum of the vector spaces of its multihomogeneous elements of square-free degree. It turns out - this is the main point - that this complex of vector spaces has the structure both of a complex of $S$-modules and of a complex of $E$-modules. The modules in this complex are not free, but they have simple and functorial free resolutions. The free resolutions of the $E$-modules in the complex fit together to make a double complex, whose total complex is the minimal free resolution of ${ }_{E} M$. A similar procedure allows us to pass in the opposite direction.

The correspondence described above works, with appropriate definitions, in a more general setting, in which $E$ is replaced by one of the algebras

$$
R_{q}:=\frac{K\left\{x_{1}, \ldots, x_{n}\right\}}{\left(\left\langle x_{j} x_{i}-q x_{i} x_{j} \mid 1 \leq i<j \leq n\right\rangle+\left\langle(1-q) x_{i}^{2} \mid 1 \leq i \leq n\right\rangle\right)}
$$

where $K\left\{x_{1}, \ldots, x_{n}\right\}$ denotes the free $K$-algebra on $x_{1}, \ldots, x_{n}$, and $q \neq 0$. We leave the details of this generalization to the interested reader.

All modules and free resolutions considered will be assumed $\mathbb{Z}^{n}$-graded. We identify $\mathbb{N}^{n} \subset \mathbb{Z}^{n}$ with the set of monomials of $S$. By the support of a monomial in either $E$ or $S$, we will mean the collection of variables present in it. A squarefree monomial (or multidegree) is an element $\mathbf{a} \in\{0,1\}^{n} \subset \mathbb{N}^{n}$; so $\operatorname{supp}(\mathbf{a})=$ $\left\{x_{j} \mid a_{j} \neq 0\right\}$.

Modules with square-free presentation. The following result is due to Bruns and Herzog BH2, Theorem $3.1 \mathrm{a})]$ :

Proposition 5.1. Let $\Gamma$ be any set of monomials of $S$ closed under taking least common multiples. If $M$ is an $S$-module with generators and relations having degrees in $\Gamma$, then all the free modules in a minimal free resolution of $M$ have degrees in $\Gamma$.

Proof. We give a new proof using Gröbner bases, which will easily extend to give Proposition [5.3 as well. Let $F \stackrel{\phi}{\rightarrow} G \rightarrow M \rightarrow 0$ be a $\mathbb{Z}^{n}$-graded free presentation with degrees of $F$ and $G$ in $\Gamma$. We may replace $F \stackrel{\phi}{\longrightarrow} G$ by a map $F^{\prime} \stackrel{\phi^{\prime}}{\longrightarrow} G$ so 
that the generators of $F^{\prime}$ map to a Gröbner basis of $\operatorname{ker}(G \rightarrow M)$ by using the Buchberger algorithm; this involves adding free generators whose degrees are the least common multiples of pairs of generators already present, and thus still in $\Gamma$. Schreyer's theorem (Eisenbud [Ei, Theorem 15.10]) shows that in the symmetric case the kernel of $\phi^{\prime}$ is generated by elements of degrees equal to the least common multiples of pairs of degrees of generators of $F^{\prime}$.

It follows as in Eisenbud Ei, Theorem 20.2] that the minimal presentation of $M$ also has degrees in $\Gamma$, and iterating this process we see that the same is true for the whole syzygy chain.

If $M$ is a square-free module in the sense above, then we say that the square-free part of $M$ is the module obtained by factoring out all the homogeneous elements of $M$ with non-square-free degrees. Thus, for example, the square-free part of $S$ itself is the factor ring $R:=S /\left(x_{1}^{2}, \ldots, x_{n}^{2}\right)$. More generally, if $\mathbf{a}$ is any square-free monomial, then $S(-\mathbf{a})$ has square-free part $R / \operatorname{supp}(\mathbf{a})(-\mathbf{a})$.

Corollary 5.2. If $M$ is a square-free module over $S$, then the square-free part of $M$ admits a resolution by direct sums of modules of the form $R / \operatorname{supp}(\mathbf{a})(-\mathbf{a})$.

An analogous result also holds over $E$ :

Proposition 5.3. If $M$ is a module over $E$ whose generators and relations have square-free degrees, then the square-free part of $M$ admits a finite resolution by modules of the form $E_{\mathbf{a}}:=E / \operatorname{supp}(\mathbf{a})(-\mathbf{a})$.

Proof. Because the generators and relations of $M$ have square-free degrees, we may write $M$ as the cokernel of a map (always $\mathbb{Z}^{n}$-homogeneous) between finite direct sums of modules of the form $E_{\mathbf{a}}$, and it thus suffices to show that the kernel of such a map is generated in square-free degrees. Using Gröbner bases we may reduce as above to the monomial case. Exactly as in Eisenbud [Ei, Lemma 15.1], one shows that all the relations among monomials are generated by those determined by the fact that any monomial $\mathbf{a}$ is annihilated by the variables in the support of $\mathbf{a}$, and the two-at-a-time relations coming from the least common multiples ("divided Koszul relations"). The desired result follows.

The common subcategory. The category of modules over $E$ and the category of modules over $R=S /\left(x_{1}^{2}, \ldots, x_{n}^{2}\right)$ have much in common. We make one such connection precise as follows:

Let $\mathbf{a}$ and $\mathbf{b}$ be two monomials in $E$ such that $\operatorname{supp}(\mathbf{a}) \subseteq \operatorname{supp}(\mathbf{b})$, and let $E_{\mathbf{a}}$ and $E_{\mathbf{b}}$ be the cyclic $E$-submodules generated by these monomials. The natural inclusion $E_{\mathbf{b}} \subseteq E_{\mathbf{a}} \subseteq E$ induces a functorial commutative diagram

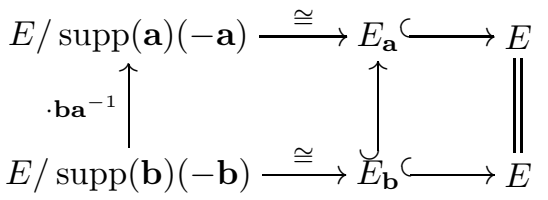

where the horizontal isomorphisms are defined by sending 1 to the distinguished generator, and the upper left monomorphism is induced by right multiplication in $E$ by $\mathbf{b a}^{-1}$, the signed exterior monomial such that $\left(\mathbf{b a}^{-1}\right) \mathbf{a}=\mathbf{b}$. 
The same commutative diagram holds if we replace $E$ by $R$. In fact, identifying square-free monomials in $E$ with the corresponding monomials in $R$ defines an equivalence of categories. More precisely:

Proposition 5.4. Let $\mathcal{A}$ denote the $K$-additive extension of the category of $\mathbb{Z}^{n}$ graded submodules of $R$, with morphisms given by inclusions, let $\mathcal{B}$ denote the $K$ additive extension of the category of $\mathbb{Z}^{n}$-graded submodules of $E$, also with morphisms given by inclusions, and let $k$ Vect be the category of $K$-vector spaces.

The above identification of square-free monomials in $E$ with those of $R$ induces an equivalence $\Psi: \mathcal{A} \rightarrow \mathcal{B}$ of categories whose restriction (via the natural forgetful functors) to the underlying $K$-vector spaces is the identity functor. In particular, the functor $\Psi$ preserves acyclic complexes.

Notice that if $\mathbf{a}$ is a square-free monomial, then the square-free part of $S(-\mathbf{a})$ is $R / \operatorname{supp}(\mathbf{a})(-\mathbf{a})=(\mathbf{a}) R$, an object of $\mathcal{A}$. Similarly, the square-free part $E_{\mathbf{a}}=$ $E / \operatorname{supp}(\mathbf{a})(-\mathbf{a})=(\mathbf{a}) E$ of $E(-\mathbf{a})$ is an object of $\mathcal{B}$.

Resolutions over $S$ and $E$. We let $\mathcal{A}_{0}$ and $\mathcal{B}_{0}$ be the additive subcategories generated by these modules and the inclusion morphisms $(\mathbf{a}) R \subset(\mathbf{b}) R$ and $(\mathbf{a}) E \subset$ (b) $E$ when a|b as monomials in $S$.

Certain free complexes over $S$ and $E$ correspond to complexes in the categories $\mathcal{A}_{0} \cong \mathcal{B}_{0}$. We describe the connection with $S$ first:

To a given $\mathbb{Z}^{n}$-graded complex $\mathbf{F}$ • of free $S$-modules

$$
\text { F. : } \quad 0 \rightarrow F_{r} \rightarrow \ldots \rightarrow F_{1} \rightarrow F_{0}
$$

with generators in square-free degrees, we associate a complex $\operatorname{sf}\left(\mathbf{F}_{\bullet}\right)$ of $R$-modules that we may regard as a complex in $\mathcal{A}_{0}$. Namely we define $\operatorname{sf}\left(\mathbf{F}_{\bullet}\right)$ as the complex of square-free degrees of $\mathbf{F} \bullet$, that is,

$$
\operatorname{sf}\left(\mathbf{F}_{\bullet}\right)_{i}=\bigoplus_{\mathbf{b} \in\{0,1\}^{n}}\left(\mathbf{F}_{i}\right)_{\mathbf{b}}
$$

for all $i$, and where the differentials are induced by the differentials of the original complex $\mathbf{F}$. . It is easy to see that sf defines a functor from the category of $\mathbb{Z}^{n}$-graded complexes of free $S$-modules to the category of complexes in $\mathcal{A}_{0}$.

It follows from Proposition 5.1 that $\mathbf{F}$ • is square-free acyclic (that is, it has no homology in square-free multidegrees) if and only if the complex $\operatorname{sf}\left(\mathbf{F}_{\bullet}\right)$ is acyclic. It is also clear that $\mathbf{F}_{\bullet}$ is minimal if and only if $\operatorname{sf}\left(\mathbf{F}_{\bullet}\right)$ is minimal.

We have proven:

Proposition 5.5. The functor $\mathrm{sf}$ is an equivalence between the category of squarefree complexes of free $S$-modules and the category of complexes in $\mathcal{A}_{0}$. It preserves minimality and acyclicity.

Now we turn to complexes over $E$. For functorial constructions, we will use the divided power algebra. If $U$ is a finite-dimensional graded vector space, we write $D_{l}(U)$ for the $l^{\text {th }}$-divided power of $U$. It is convenient to define $D_{l}(U)$ as the dual of the $l^{\text {th }}$-symmetric power of the dual space, that is, $D_{l}(U)=\left(\operatorname{Sym}_{l}\left(U^{*}\right)\right)^{*}$. The divided powers $D_{l}(U)$ have "diagonal" maps $D_{l+1}(U) \rightarrow D_{l}(U) \otimes U$, which are the monomorphisms dual to the surjective natural multiplication map in the symmetric algebra $\operatorname{Sym}_{l}\left(U^{*}\right) \otimes U^{*} \rightarrow \operatorname{Sym}_{l+1}\left(U^{*}\right)$.

We can now go from complexes in the category $\mathcal{B}_{0}$ to free complexes over $E$ using the Cartan Resolution. 
Proposition 5.6. There exists a functor $\Phi$ from the category of complexes in $\mathcal{B}_{0}$ to the category of complexes of free modules over $E$ whose inverse is obtained by taking square-free parts. $\Phi$ preserves acyclicity and minimality. Applied to an acyclic complex in $\mathcal{B}_{0}$ with homology $M$, the functor $\Phi$ provides an $E$-free resolution of $M$.

Proof. We first define $\Phi$ on modules in $\mathcal{B}_{0}$. It associates, to a cyclic module $E_{\mathbf{a}} \cong$ $E / \operatorname{supp}(\mathbf{a})(-\mathbf{a})$, the (resolution) $\Phi\left(E_{\mathbf{a}}\right):=D\left(L_{\mathbf{a}}\right) \otimes E$, where

$$
L_{\mathbf{a}}:=\bigoplus_{x_{i} \in \operatorname{supp}(\mathbf{a})} k\left(-\mathbf{e}_{i}\right)
$$

is the $\mathbb{Z}^{n}$-graded subspace of $V$ spanned by $\operatorname{supp}(\mathbf{a})$, and whose differentials are induced by the diagonals followed by multiplication in $E$.

More precisely, $\Phi\left(E_{\mathbf{a}}\right)$ is the complex

$$
\Phi\left(E_{\mathbf{a}}\right): \ldots \rightarrow D_{2}\left(L_{\mathbf{a}}\right) \otimes E(-\mathbf{a}) \rightarrow L_{\mathbf{a}} \otimes E(-\mathbf{a}) \rightarrow E(-\mathbf{a}),
$$

which is a minimal free resolution of the cyclic module $E_{\mathbf{a}}$. We see at once that $E_{\mathbf{a}}$ is the square-free part of $\Phi\left(E_{\mathbf{a}}\right)$.

If $\mathbf{a}$ and $\mathbf{b}$ are two monomials in $E$ such that $\operatorname{supp}(\mathbf{a}) \subseteq \operatorname{supp}(\mathbf{b})$, then

$$
D\left(L_{\mathbf{b}}\right) \otimes E(-\mathbf{b}) \stackrel{\pi \otimes\left(\cdot \mathbf{b a}^{-1}\right)}{\longrightarrow} D\left(L_{\mathbf{a}}\right) \otimes E(-\mathbf{a}),
$$

where $\pi$, the map induced to divided powers by the canonical projection $\pi: L_{\mathbf{b}} \rightarrow$ $L_{\mathbf{a}}$, is a morphism of chain complexes lifting the inclusion $E_{\mathbf{b}} \subseteq E_{\mathbf{a}} \subseteq E$.

Given a complex $\mathbf{F}$ • in $\mathcal{B}$, we may apply $\Phi$ to obtain a double complex of free $E$ modules, and we set $\Phi\left(\mathbf{F}_{\bullet}\right)$ to be the total complex of this double complex. Because of the way $\Phi$ is defined on each object of $\mathcal{B}_{0}$, this functor preserves minimality. The spectral sequences of the double complex shows that it also preserves acyclicity.

As Aramova, Avramov, Herzog [AAH] and Römer [Rö] observe, the existence of such a construction shows that an $S$-module $M$ has a linear free resolution over $S$ if and only if the corresponding $E$-module has a linear free resolution over $E$. Our version of the construction also "explains" these authors' formula for Betti numbers:

Corollary 5.7. The following equality holds among Poincaré series:

$$
\sum_{i=0}^{\infty} \sum_{\mathbf{a} \in \mathbb{N}^{n}} \beta_{i, \mathbf{a}}^{E}\left({ }_{E} M\right) t^{i} \mathbf{u}^{\mathbf{a}}=\sum_{i=0}^{\infty} \sum_{\mathbf{a} \in \mathbb{N}^{n}} \beta_{i, \mathbf{a}}^{S}\left({ }_{S} M\right) \frac{t^{i} \mathbf{u}^{\mathbf{a}}}{\prod_{j \in \operatorname{supp}(\mathbf{a})}\left(1-t u_{j}\right)},
$$

where $\beta_{i, \mathbf{a}}^{E}\left({ }_{E} M\right)$ denotes the dimension of the degree a part of $\operatorname{Tor}_{i}^{E}(M, K)$, and similarly for $\beta_{i, \mathbf{a}}^{S}\left({ }_{S} M\right)$.

\section{REFERENCES}

[AAH] A. Aramova, L. A. Avramov, J. Herzog: Resolutions of monomial ideals and cohomology over exterior algebras, Trans. Amer. Math. Soc. 352 (2000), no. 2, 579-594. MR 2000c:13021

[AHH] A. Aramova, J. Herzog, T. Hibi: Squarefree lexsegment ideals, Math. Z. 228, (1998), 353-378. MR 99h:13013

[BCP] D. Bayer, H. Charalambous, S. Popescu: Extremal Betti Numbers and Applications to Monomial Ideals, J. Algebra 221, (1999), 497-512. MR 2001a:13020

[BP] L. J. Billera, J. S. Provan: Decompositions of simplicial complexes related to diameters of convex polyhedra, Math. Oper. Res. 5, (1980), 576-594. MR 82c:52010 
[Bjö1] A. Björner: On the homology of geometric lattices, Algebra Univ. 14, (1982), 107-128. MR 83d:05029

[Bjö2] A. Björner: The homology and shellability of matroids and geometric lattices, Chapter 7 of Matroid Applications, ed. Neil White, 226-283, Encyclopedia Math. Appl., 40, Cambridge Univ. Press, Cambridge, 1992. MR 94a:52030

[BZ] A. Björner, G. Ziegler: Combinatorial stratification of complex arrangements, J. Amer. Math. Soc. 5, (1992), no. 1, 105-149. MR 92k:52022

[Bo] B. Bollobás: Modern Graph Theory, Graduate Texts in Mathematics 184, Springer-Verlag, New York, 1998. MR 99h:05001

[BH1] W. Bruns, J. Herzog: Cohen-Macaulay Rings, Cambridge Studies in Advanced Mathematics, 39, Cambridge University Press, 1993. MR 95h:13020

[BH2] W. Bruns, J. Herzog: On multigraded resolutions, Math. Proc. Cambridge Philos. Soc., 118, (1995), 245-257. MR 96g:13013

[CS] D. Cohen, A. Suciu: Characteristic varieties of arrangements, Math. Proc. Cambridge Philos. Soc. 127 (1999), no. 1, 33-53. MR 2000m:32036

[Cr] H. Crapo: A higher invariant for matroids, J. of Combinatorial Theory 2, (1967), 406-417. MR 35:6579

[ER] J. Eagon, V. Reiner: Resolutions of Stanley-Reisner rings and Alexander duality, J. Pure Appl. Algebra 130, (1998), no. 3, 265-275. MR 99h:13017

[Ei] D. Eisenbud: Commutative Algebra with a View Toward Algebraic Geometry, SpringerVerlag, New York, 1995. MR 97a:13001

[ES] D. Eisenbud, F.-O. Schreyer: Sheaf Cohomology and Free Resolutions over Exterior Algebras, preprint math.AG/0005055.

[ESV] H. Esnault, V. Schechtman, E. Viehweg: Cohomology of local systems on the complement of hyperplanes, Invent. Math. 109 (1992), no. 3, 557-561. MR 93g:32051

[Fa] M. Falk: Arrangements and cohomology, Ann. Comb. 1 (1997), no. 2, 135-157. MR 99g:52017

[GS] D. Grayson, M. Stillman: Macaulay2, a software system devoted to supporting research in algebraic geometry and commutative algebra. Contact the authors, or download from ftp://ftp.math.uiuc.edu/Macaulay2.

[Ho1] M. Hochster: Rings of invariants of tori, Cohen-Macaulay rings generated by monomials, and polytopes, Ann. of Math. 96, (1972), 318-337. MR 46:3511

[Ho2] M. Hochster: Cohen-Macaulay rings, combinatorics and simplicial complexes, in Ring theory II, McDonald, B. R. and Morris, R. A. (eds.), Lecture Notes in Pure and Appl. Math. 26, Marcel Dekker, 1977. MR 56:376

[LY] A. Libgober, S. Yuzvinsky: Cohomology of the Orlik-Solomon algebras and local systems, Compositio Math. 121 (2000), no. 3, 337-361. MR 2001j:52032

[MT] W. Massey, L. Traldi: On a conjecture of K. Murasugi, Pacific J. Math. 124 (1986), no. 1, 193-213. MR 87k:57008

[MS] D. Matei, A. Suciu: Cohomology rings and nilpotent quotients of real and complex arrangements, Singularities and Arrangements, Sapporo-Tokyo 1998, Advanced Studies in Pure Mathematics 27 (2000), 185-215. MR 2002b:32045

[Mi] J. Milnor: Isotopy of links, in Algebraic geometry and topology. A symposium in honor of S. Lefschetz, pp. 280-306. Princeton University Press, Princeton, N. J., 1957. MR 19:1070c

$[\mathrm{Mu}]$ M. Mustaţă: Local Cohomology at Monomial Ideals, in "Symbolic computation in algebra, analysis, and geometry (Berkeley, CA, 1998)", J. Symbolic Comput. 29 (2000), no. 4-5, 709-720. MR 2001i:13020

[OS] P. Orlik, L. Solomon: Combinatorics and topology of complements of hyperplanes, Invent. Math. 56, (1980), 167-189. MR 81e:32015

[OT] P. Orlik, H. Terao: Arrangements of hyperplanes, Grundlehren der Mathematischen Wissenschaften 300, Springer-Verlag, Berlin, 1992. MR 94e:52014

[Pr] J. S. Provan: Decompositions, shellings, and diameters of simplicial complexes and convex polyhedra, Thesis, Cornell Univ., Ithaca, NY, 1977.

[Rö] T. Römer: Generalized Alexander Duality and Applications, Osaka J. Math. 38 (2001), 469-485. MR 2002c: 13029

[STV] V. Schechtman, H. Terao, A. Varchenko: Local systems over complements of hyperplanes and the Kac-Kazhdan conditions for singular vectors, J. Pure Appl. Algebra 100, (1995), 93-102. MR 96j:32047 
[St1] R. Stanley: Combinatorics and Commutative Algebra, Second edition, Progress in Math. 41, Birkhäuser, 1996. MR 98h:05001

[St2] R. Stanley: Cohen-Macaulay rings and constructible polytopes, Bull. Amer. Math. Soc. 81, (1975), 133-135. MR 51:486

[Te] N. Terai: Generalization of Eagon-Reiner theorem and $h$-vectors of graded rings, preprint 1997.

[Ya] K. Yanagawa: Alexander duality for Stanley-Reisner rings and square-free $\mathbf{N}^{n}$-graded modules, J. Algebra 225 (2000), no. 2, 630-645. MR 2000m:13036

[Yu] S. Yuzvinsky: Cohomology of the Brieskorn-Orlik-Solomon algebras, Comm. Algebra 23, (1995), 5339-5354. MR 97a:52023

[Zi] G. Ziegler: On the difference between real and complex arrangements, Math. Z. 212 (1993), no. 1, 1-11. MR 94f:52017

Department of Mathematics, University of California Berkeley, Berkeley, CaliforNIA 94720

E-mail address: de@msri.org

Department of Mathematics, SUny at Stony Brook, Stony Brook, New York 11794

E-mail address: sorin@math.sunysb.edu

Department of Mathematics, University of Oregon, Eugene, Oregon 97403

E-mail address: yuz@math.uoregon.edu 